\title{
Wetting, roughness and flow boundary conditions
}

\author{
Olga I Vinogradova ${ }^{1,2}$ and Aleksey V Belyaev $^{1,3}$ \\ ${ }^{1}$ A N Frumkin Institute of Physical Chemistry and Electrochemistry, Russian Academy of \\ Sciences, 31 Leninsky Prospect, 119991 Moscow, Russia \\ 2 ITMC and DWI, RWTH Aachen, Pauwelsstraße 8, 52056 Aachen, Germany \\ ${ }^{3}$ Physics Department, M V Lomonosov Moscow State University, 119991 Moscow, Russia \\ E-mail: oivinograd@yahoo.com
}

Received 31 May 2010, in final form 18 October 2010

Published 20 April 2011

Online at stacks.iop.org/JPhysCM/23/184104

\begin{abstract}
We discuss how the wettability and roughness of a solid impacts its hydrodynamic properties.

We see in particular that hydrophobic slippage can be dramatically affected by the presence of roughness. Owing to the development of refined methods for setting very well controlled micro- or nanotextures on a solid, these effects are being exploited to induce novel hydrodynamic properties, such as giant interfacial slip, superfluidity, mixing and low hydrodynamic drag, that could not be achieved without roughness.
\end{abstract}

(Some figures in this article are in colour only in the electronic version)

\section{Introduction}

Fluid mechanics is one of the oldest and most useful of the 'exact' sciences. For hundreds of years it has relied upon the no-slip boundary condition at a solid-liquid interface that was applied successfully to model many macroscopic experiments [1]. However, the problem is not that simple and has been revisited in recent years. One reason for such a strong interest in this 'old' problem is purely fundamental. The noslip boundary condition is an assumption that cannot be derived from first principles even for a molecularly smooth hydrophilic (the contact angle, fixed by the chemical nature of a solid, lies between $0^{\circ}$ and $90^{\circ}$ ) surface. Therefore, the success of the no-slip postulate may not always reflect its accuracy but in fact rather the insensitivity of the experiment. Another reason for current interest in flow boundary conditions lies in the potential applications in many areas of engineering and applied science that deal with small size systems, including micro- and nanofluidics [2], flow in porous media, friction and lubrication, and biological fluids. The driving and mixing of liquids when the channel size decreases represent very difficult problems [3]. There is therefore a great hope that changes in hydrodynamic behavior can be modified by interfacial phenomena on the flow. For example, even ideal solids, which are both flat and chemically homogeneous, can have a contact angle that exceeds $90^{\circ}$ (the hydrophobic case). This can modify the hydrodynamic boundary conditions, as has already been shown in early work [4]. Furthermore, solids are not ideal but rough. This can further change, and quite dramatically, the boundary conditions. It is of course interesting and useful to show how the defects or pores of the solids modify the conditions. But today, the question has slightly shifted. Thanks to techniques coming from microelectronics, we are able to elaborate substrates whose surfaces are patterned (often at the micro- and nanometer scale) in a very well controlled way, thus providing properties (e.g. optical or electrical) that the solid did not have when flat or slightly disordered. A texture affects the wettability and boundary conditions on a substrate, and can induce unique properties that the material could not have without these micro- and nanostructures. In particular, in the case of super-hydrophobic solids, which are generated by a combination of surface chemistry and patterns, roughness can dramatically lower the ability of drops to stick, leading to the remarkable mobility of liquids. At the macroscopic scale this renders them 'self-cleaning' and causes droplets to roll (rather than slide) under gravity and rebound (rather than spread) upon impact instead of spreading [5]. At the smaller scale, reduced wall friction and a superlubricating potential are almost certainly associated with the breakdown of the no-slip hypothesis.

In this paper we concentrate on the understanding and expectations for the fluid-solid boundary conditions in different situations where hydrophobicity and roughness impact the flow properties. After introducing the terminology, 

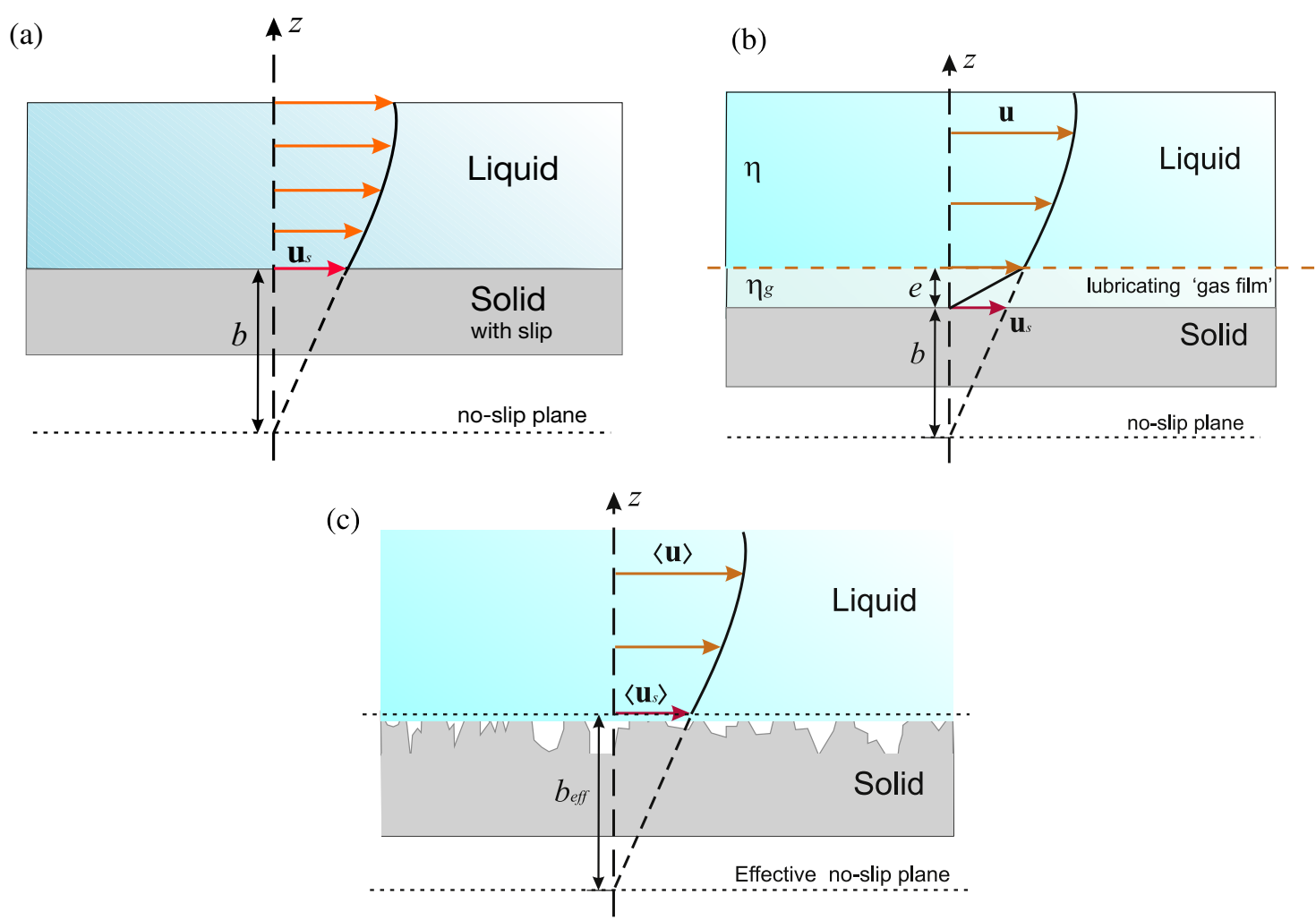

Figure 1. Schematic representation of the definition of intrinsic (a), apparent (b) and effective (c) slip lengths.

and describing new developments and instruments that give the possibility of investigating fluid behavior at the micro- and nanoscale, in the following section we present results obtained for smooth surfaces, by highlighting the role of wettability. Then follows the results for rough hydrophilic and, especially, hydrophobic surfaces. In the latter case we show, and this is perhaps the main message of our paper, how roughness can enhance hydrodynamic slip and thus the efficiency of transport phenomena.

\section{Terminology}

We will refer to slip as being any situation where the value of the tangential component of velocity appears to be different from that of the solid surface. The simplest possible relation assumes that the tangential force per unit area exerted on the solid surface is proportional to the slip velocity. Combining this with the constitutive equation for the bulk Newtonian fluid one gets the so-called (scalar) Navier boundary condition

$$
u_{\mathrm{s}}=b \frac{\partial u}{\partial z}
$$

where $u_{\mathrm{s}}$ is the (tangential) slip velocity at the wall, $\partial u / \partial z$ the local shear rate and $b$ the slip length. This slip length represents a distance inside the solid to which the velocity has to be extrapolated to reach zero. The standard no-slip boundary condition corresponds to $b=0$, and the shear-free boundary condition corresponds to $b \rightarrow \infty$ [6]. In the most common situation $b$ is finite (a partial slip) and associated with the positive slip velocity. It can, however, be negative, although in this case it would not have a long-range effect on the flow [7]. Obviously the control of slip lengths is of major importance for flow at an interface and in a confined geometry. It would be useful to distinguish between three different situations for a boundary slip since the dynamics of fluids at the interface introduce various length scales.

Molecular (or intrinsic) slip, which allows liquid molecules to slip directly over a solid surface (figure 1(a)). Such a situation is not of main concern here since molecular slip cannot lead to a large $b[4,8,9]$ and its calculations require a molecular consideration of the interface region. In particular, recent molecular dynamics simulations predicted a molecular $b$ below $10 \mathrm{~nm}$ for realistic contact angles $[10,11]$. Therefore, it is impossible to benefit from such a slip in a larger scale applications.

The intrinsic boundary condition may be rather different from what is probed in flow experiments at larger length scale. It has been proposed [6] to describe the interfacial region as a lubricating 'gas film' of thickness $e$ of viscosity $\eta_{\mathrm{g}}$ different from its bulk value $\eta$. A straightforward calculations give apparent slip (figure $1(\mathrm{~b})$ )

$$
b=e\left(\frac{\eta}{\eta_{\mathrm{g}}}-1\right) \simeq e \frac{\eta}{\eta_{\mathrm{g}}} .
$$

This represents the so-called 'gas cushion model' of hydrophobic slippage [6], which has a clear microscopic foundation in terms of a prewetting transition [12]. Being a schematic representation of a depletion close to a wall [13], 
this model provides a useful insight into the sensitivity of the interfacial transport to the structure of an interface. Similarly, electrokinetic flow displays apparent slip.

Another situation is that of effective slip, $b_{\text {eff }}$, which refers to a situation where slippage at a complex heterogeneous surface is evaluated by averaging of a flow over the length scale of the experimental configuration (e.g. a channel etc) $[3,14-16]$. In other words, rather than trying to solve equations of motion at the scale of the individual corrugation or pattern, it is appropriate to consider the 'macroscale' fluid motion (on the scale larger than the pattern characteristic length or the thickness of the channel) by using effective boundary conditions that can be applied at the imaginary smooth surface. Such an effective condition mimics the actual one along the true heterogeneous surface. It fully characterizes the flow at the real surface and can be used to solve complex hydrodynamic problems without tedious calculations. Such an approach is supported by a statistical diffusion arguments (being treated as an example of commonly used OnsagerCasimir relations for non-equilibrium linear response) [14] theory of heterogeneous porous materials [15], and has been justified for the case of Stokes flow over a broad class of surfaces [16]. For anisotropic textures $b_{\text {eff }}$ depends on the flow direction and is generally a tensor [14]. Effective slip also depends on the interplay between typical length scales of the system as we will see below. Well-known examples of such a heterogeneous system include composite super-hydrophobic (Cassie) surfaces, where a gas layer is stabilized with a rough wall texture (figure 1(c)). For these surfaces effective slip lengths are often very large compared with the value on flat solids, similar to what has been observed for wetting, where the contact angle can be dramatically enhanced when the surface is rough and heterogeneous [17].

\section{Experimental methods}

The experimental challenge has generated considerable progress in the experimental tools for investigating flow boundary conditions, using the most recent developments in optics and scanning probe techniques. A great variability still exists in the results of slip experiments so it is important first to consider the different experimental methods used to measure slip. Two broad classes of experimental approaches have been used so far: indirect and direct (local) methods.

High-speed force measurements can be performed with the SFA (surface forces apparatus) [18-20] or AFM (atomic force microscope) [21]. In particular, in the drainage method $[18,21]$ the end of the spring away from the attached sphere is driven toward the (fixed) plane with a constant driving speed (as shown in figure 2). The sphere itself, however, does not move at a constant speed, so that the spring is deflected as a result of both the surface force (which should be measured separately) and the hydrodynamic forces. The solution of the (differential) equation of motion allows one to deduce a drag force, with the subsequent comparison with a theory of a film drainage [6, 22]. This approach, being extremely accurate at the nanoscale, does not provide visualization of the flow profile, so these measurements are identified as indirect.

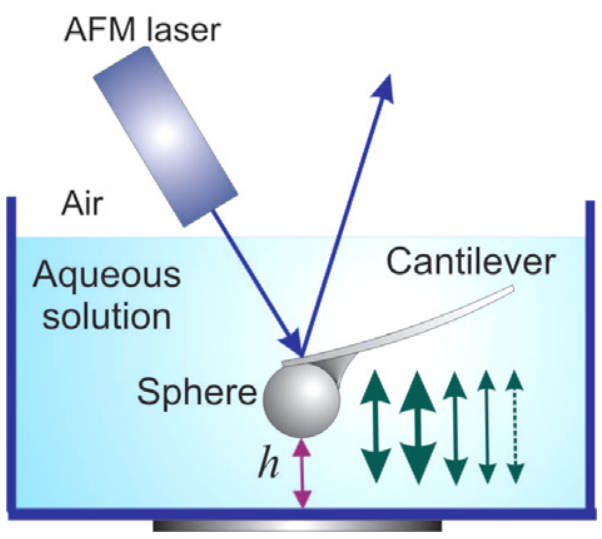

Figure 2. Schematic of the dynamic AFM force experiment.

Direct approaches to flow profiling, or velocimetry, take advantage of various optics to monitor tracer particles. These methods include TIR-FRAP (total internal reflection fluorescence recovery after photo-bleaching) [23], $\mu$-PIV [24, 25] (particle image velocimetry), TIRV (total internal reflection velocimetry) [26], EW $\mu$-PIV (evanescent wave micro-particle image velocimetry) [27], and multilayer nano-particle image velocimetry (nPIV) [28]. Their accuracy is normally much lower than that of force methods due to relatively low optical resolution, system noise due to polydispersity of tracers and difficulties in decoupling flow from diffusion (the tracer distribution in the flow field is affected by Taylor dispersion [29]). As a consequence, it has been always expected that a slippage of the order of a few tens of nanometers cannot be detected by a velocimetry technique. However, recently, direct high-precision measurements at the nanoscale have been performed with a new optical technique, based on a DF-FCS (double-focus spatial fluorescence crosscorrelation) [30, 29] (as is schematically shown in figure 3). As the fluorescence tracers flow along the channel they cross the two foci in turn, producing two time-resolved fluorescence intensities, $I_{1}(t)$ and $I_{2}(t)$, recorded independently. The time cross-correlation function can be calculated and typically exhibits a local maximum. The position of this maximum $\tau_{M}$ is characteristic of the local velocity of the tracers. Another example of high resolution applications of FCS consists in the determination of the average transverse diffusion coefficient to probe slippage [31]. Since FCS methods allow consideration of $N \sim 10^{6}$ particles, this gives a satisfactory signal to noise ratio $\sqrt{N}$ of the order $10^{3}$, providing extremely good resolution as compared with other direct velocimetry methods. Coupling this with TIRF [32], which allows the measurements of the distance of tracers from the wall through the exponential decay of an evanescent wave, should further improve the accuracy of the approach.

Many experiments have been performed on the subject, with somewhat contradictory results. Experimental work focused mostly on bare (smooth) surfaces, more recent investigations have turned towards rough and structured surfaces, in particular super-hydrophobic surfaces [33]. We refer the reader to comprehensive review articles [8, 34] for detailed accounts of the early experimental work. Below we 


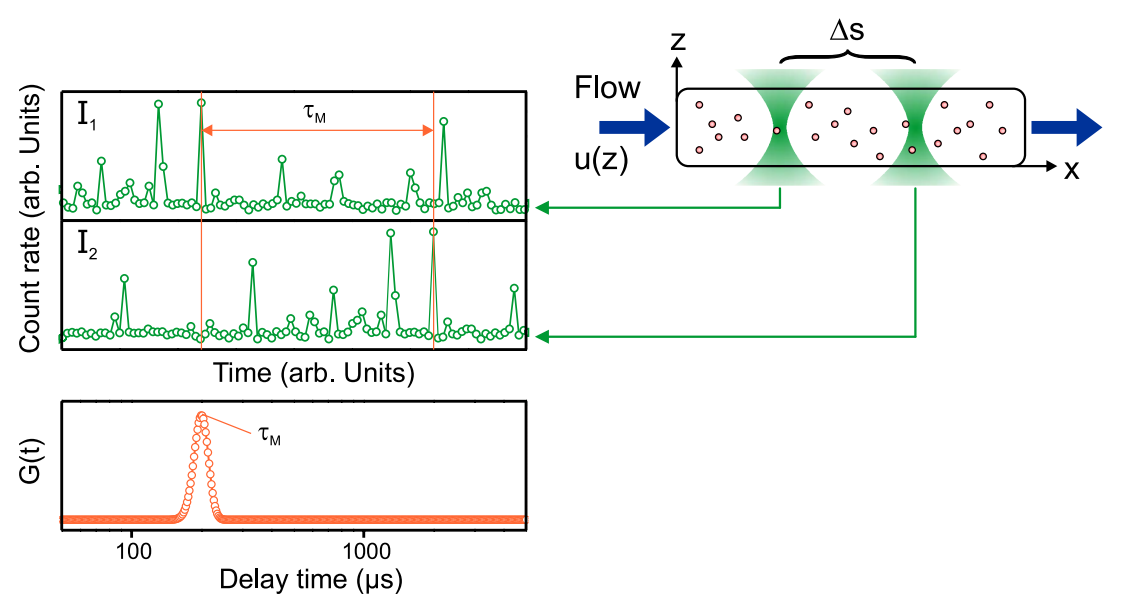

Figure 3. Schematics of the double-focus spatial fluorescence cross-correlation method. Two laser foci are located along the $x$ axis, separated by a distance of a few micrometers. They independently record the time-resolved fluorescence intensities $I_{1}(t)$ and $I_{2}(t)$. The forward cross-correlation of these two signals yields $G(t)$. Two foci are scanned simultaneously along the $z$ axis to probe the velocity profile $u(z)$.

mention only what we believe is the most relevant recent contribution to the subject of flow past 'simple' smooth surfaces, which clarified the situation, highlighted the reasons for the existing controversies and resolved apparent paradoxes. We focus, however, more on the implication of micro- and nanostructuring on fluidic transport, which is still in its infancy and remains to be explored.

\section{Smooth surfaces: slippage versus wetting}

From the theoretical $[9,35]$ and simulation $[11,36]$ point of view, slippage should not appear on a hydrophilic surface, except probably at a very high shear rate [37]. A slip length of the order of a hundred nanometers or smaller is, however, expected for a hydrophobic surface $[6,9,12,38]$. On the experimental side, no consensus was achieved until recently. While some experimental data were consistent with the theoretical expectations both for hydrophilic and hydrophobic surfaces $[19,21]$, some other reports completely escaped from this picture with both qualitative (slippage over hydrophilic surfaces, shear rate dependent slippage, rate thresholds for slip, etc) and quantitative (slip length of several micrometers) discrepancies (for a review see [8]). More recent experiments, performed with various new experimental methods, finally concluded that water does not slip on smooth hydrophilic surfaces, and develops a slip only on hydrophobic surfaces [29, 31, 39-42]. One can therefore conclude that a concept of hydrophobic slippage is now widely accepted.

An important issue is the amplitude of hydrophobic slip. The observed slip length reached the range 20-100 nm, which is above predictions of the models of molecular slip [10, 43]. This suggests the apparent slip, such as the 'gas cushion model', equation (2). Water glides on air, owing to the large viscosity ratio between water and air (typically a factor of 50). Experimental values of $b$ suggest that the thickness of this 'layer' is below $2 \mathrm{~nm}$. A modification of this scenario would be a nanobubble coated surface [44-47]. Another important conclusion is that it is impossible to benefit from such a nanometric slip at separations $\mathrm{O}(\mu \mathrm{m})$ and larger, i.e. in microfluidic applications. This is why in the discussion of super-hydrophobic slippage below we often ignore a slip past hydrophobic solids. However, a hydrophobic slippage is likely to be of major importance in nanochannels (highly confined hydrophobic pores, biochannels, etc), where ordinary Poiseuille flow is fully suppressed.

\section{Rough surfaces}

Only a very few solids are molecularly smooth. Most of them are naturally rough, often at a micro- and nanoscale, due to their structure, methods of preparation, various coatings, etc. These surfaces are very often in the Wenzel (impaled) state, where the solid-liquid interface has the same area as the solid surface (figure 4(a)). However, even for rough hydrophilic Wenzel surfaces the situation is not very clear, and opposing experimental conclusions have been made: one is that roughness generates extremely large slip [48], whereas another is that it decreases the degree of slippage [49, 50]. More recent experimental data suggests that the description of flow near rough surfaces has to be corrected, but for a separation, not slip [39]. The theoretical description of such a flow represents a difficult, nearly insurmountable, problem. It has been solved only approximately, and only for a case of the periodic roughness and far-field flow with a conclusion that it may be possible to approximate the actual surface by a smooth one with the slip boundary condition [51-53].

This issue was recently resolved in a LB (lattice Boltzmann) simulation study [36], where the hydrodynamic interaction between a smooth sphere of radius $R$ and a randomly rough plane was studied (as shown in figure 5). Beside its significance as a geometry of SFA/AFM dynamic force experiments, this allows one to explore both far- and near-field flows in a single 'experiment'. The 'measured' hydrodynamic force was smaller than predicted for two smooth surfaces (with the separation defined at the top of the asperities) when the standard no-slip boundary conditions were used in the calculation. Moreover, at small separations the force was even weaker and showed different asymptotics 


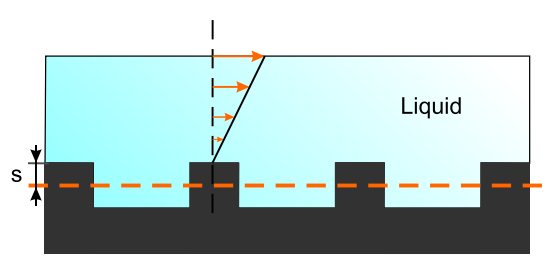

(a)

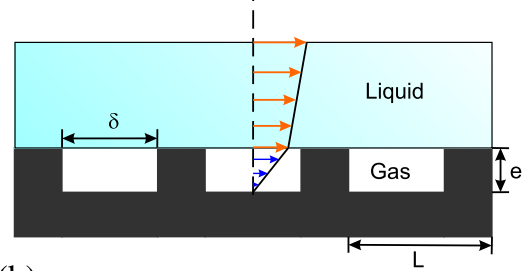

(b)

Figure 4. Schematic representation of the (a) Wenzel and (b) Cassie pictures with the local flow profiles at the gas and solid areas.

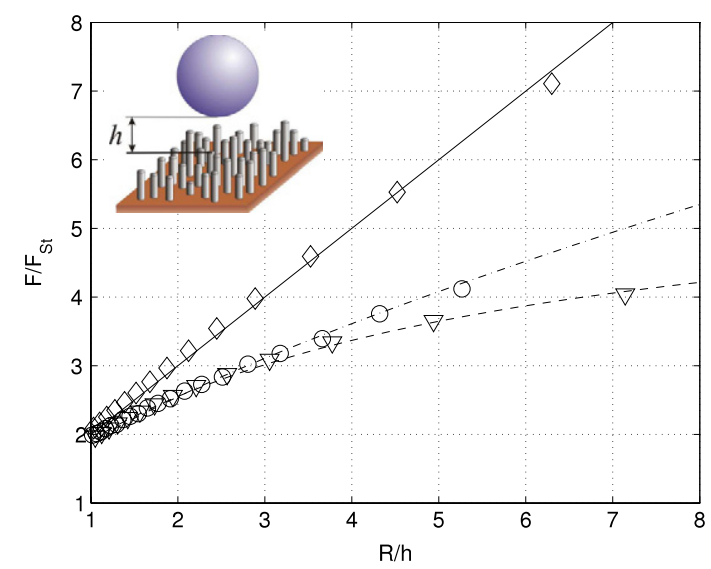

Figure 5. Hydrodynamic force acting on a hydrophilic sphere of radius $R$ approaching a smooth hydrophilic (diamonds), smooth hydrophobic (circles) and randomly rough hydrophilic (triangles) wall with $\phi_{2}=4 \%$ (adapted from [36]). Here $F_{\mathrm{St}}=6 \pi \eta R U$ is the Stokes drag. The separation $h$ is defined on top of the surface roughness, as shown in the inset. Simulation results (symbols) compared with theoretical curves: $F / F_{\mathrm{St}}=1+9 R /(8 h)$ (solid), $F / F_{\mathrm{St}}=1+9 R f^{*} /(8 h)$ with $f^{*}=f(b / h)$ taken from [7] (dash-dotted) and $F / F_{\mathrm{St}}=1+9 R /(8[h+s])$ (dashed). Values of $b$ and $s$ were determined by fitting the simulation data.

than those expected if one invokes slippage at the smooth fluid-solid interfaces. This can only be explained by the model of a no-slip wall, located at an intermediate position (controlled by the density of roughness elements) between the top and bottom of the asperities (illustrated by the dashed line figure 4(a)). Calculations based on this model provided an excellent description of the simulation data (figure 5).

\section{Super-hydrophobicity and effective hydrodynamic slippage}

On hydrophobic solids, the situation is different from that on hydrophilic solids. If the solid is rough enough, we do not expect that the liquid will conform to the solid surface, as assumed in the Wenzel or impaled state. Rather, air pockets should form below the liquid, provided that the energetic cost associated with all the corresponding liquid-vapor interfaces is smaller than the energy gained not to follow the solid [5]. This is the so-called Cassie or fakir state. Hydrophobic Cassie materials generate large contact angles and small hysteresis, ideal conditions for making water drops very mobile. It is natural to expect a large effective slip in a Cassie situation.
Indeed, taking into account that the variation of the texture height, $e$, is in the typical interval $0.1-10 \mu \mathrm{m}$, according to equation (2) we get $b=5-500 \mu \mathrm{m}$ at the gas area. The composite nature of the texture requires regions of very low slip (or no slip) in direct contact with the liquid, so the effective slip length of the surface, $b_{\text {eff }}$, is smaller than $b$. Still, one can expect that a rational design of such a texture could lead to a large values of $b_{\text {eff. }}$. Below we make these arguments more quantitative.

We will examine an idealized super-hydrophobic surface in the Cassie state, sketched in figure 4(b), where a liquid slab lies on top of the surface roughness. The liquid-gas interface is assumed to be flat with no meniscus curvature, so that the modeled super-hydrophobic surface appears as perfectly smooth with a pattern of boundary conditions. In the simplified description the latter are taken as no-slip $\left(b_{1}=0\right)$ over solidliquid areas and as partial slip $\left(b_{2}=b\right)$ over gas-liquid regions (as we have shown above, $b_{1}$ is of the orders of tens of nanometers, so that we could neglect it since $b_{2}$ is of the order of tens of micrometers). We denote as $\delta$ the typical length scale of gas-liquid areas. The fraction of solid-liquid areas will be denoted $\phi_{1}=(L-\delta) / L$, and of gas-liquid area $\phi_{2}=1-\phi_{1}=$ $\delta / L$. Overall, the description of a super-hydrophobic surface we use is similar to those considered in [15, 54-57]. In this idealization, some assumptions may have a possible influence on the friction properties and, therefore, a hydrodynamic force. First, by assuming a flat interface, we have neglected an additional mechanism for a dissipation connected with the meniscus curvature [58-60]. Second, we ignore a possible transition towards the impaled (Wenzel) state that can be provoked by additional pressure in the liquid phase [61, 62].

Finally, for the sake of brevity we focus below only on the canonical microfluidic geometry where the fluid is confined between flat plates, and only on the asymmetric case, where one (upper) surface is smooth hydrophilic and the other (lower) represents a super-hydrophobic wall in the Cassie state. Such a configuration is relevant for various setups, where the alignment of opposite textures is inconvenient or difficult. We also restrict the discussion to a pressure-driven flow governed by the Stokes equations

$$
\eta \nabla^{2} \mathbf{u}=\nabla p, \quad \nabla \cdot \mathbf{u}=0
$$

where $\mathbf{u}$ is the velocity vector, and $p$ is pressure. Extensions of our analysis to study other configuration geometries and types of flow would be straightforward. 


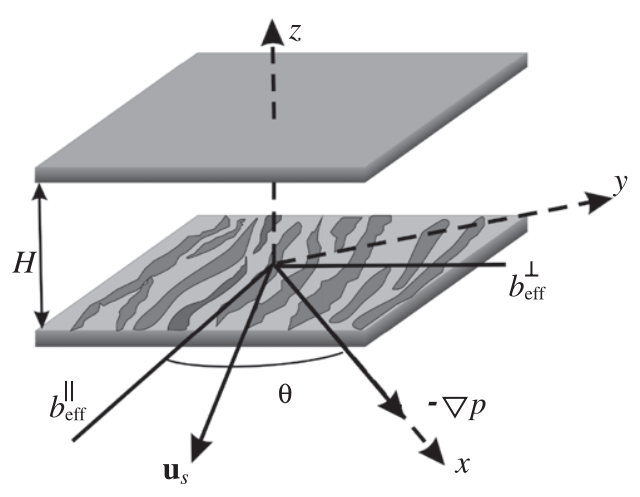

Figure 6. Sketch of a flat channel of thickness $H$, with notation for directions along the plates. One wall represents an anisotropic super-hydrophobic texture.

\subsection{Anisotropic surfaces}

Many natural and synthetic textures are isotropic. However, it can be interesting to design directional structures, such as arrays of parallel grooves or microwrinkles, that consequently generate anisotropic effective slip in the Cassie regime. The hydrodynamic slippage is quite different along and perpendicular to the grooves. Axial motion is preferred, and such designs are appropriate when liquid must be guided. There are examples of such patterns in nature, such as the wings of butterflies or the legs of water striders.

The flow past such surfaces becomes misaligned with the pressure gradient and has been analyzed in a number of studies $[63,64]$. Such phenomena have motivated a tensorial version of (1), as discussed in [3, 14]:

$$
\left\langle\left. u_{i}\right|_{A}\right\rangle=\sum_{j, k} b_{i j}^{\mathrm{eff}} n_{k}\left\langle\left.\frac{\partial u_{j}}{\partial x_{k}}\right|_{A}\right\rangle,
$$

where $\left\langle\left.\mathbf{u}\right|_{A}\right\rangle$ is the effective slip velocity, averaged over the surface pattern and $\mathbf{n}$ is a unit vector normal to the surface $A$. The second-rank effective slip tensor $\mathbf{b}_{\text {eff }} \equiv\left\{b_{i j}^{\text {eff }}\right\}$ characterizes the surface anisotropy and is represented by symmetric, positive definite $2 \times 2$ matrix diagonalized by a rotation:

$\mathbf{b}_{\text {eff }}=\mathbf{S}_{\theta}\left(\begin{array}{cc}b_{\mathrm{eff}}^{\|} & 0 \\ 0 & b_{\mathrm{eff}}^{\perp}\end{array}\right) \mathbf{S}_{-\theta}, \quad \mathbf{S}_{\theta}=\left(\begin{array}{cc}\cos \theta & \sin \theta \\ -\sin \theta & \cos \theta\end{array}\right)$.

As proven in [14], for all anisotropic surfaces the eigenvalues $b_{\text {eff }}^{\|}$and $b_{\text {eff }}^{\perp}$ of the slip-length tensor correspond to the fastest (greatest forward slip) and slowest (least forward slip) directions, which are always orthogonal (see figure 6).

To illustrate the calculation of the slip-length tensor, below we consider the geometry where the liquid is confined between two plates separated by a distance $H$, and one of them represents a super-hydrophobic striped wall (figure 7(a)).

6.1.1. General solution. We calculate the effective slip lengths in the eigendirections (which in this case are obviously parallel and orthogonal to the stripes), by solving the Stokes equations (3). The $x$-axis is directed along the pressure gradient $\langle\nabla p\rangle=(-\sigma, 0,0)$. Essentially, since along these orthogonal directions there are no transverse hydrodynamic couplings [14], the pressure gradient $\langle\nabla p\rangle$ coincides with the direction of slip for longitudinal and transverse stripes. We seek the solution for a velocity $\mathbf{u}$ by perturbation analysis:

$$
\mathbf{u}=\mathbf{u}_{0}+\mathbf{u}_{1},
$$

where $\mathbf{u}_{0}$ is the velocity of the Poiseuille flow, and the effective slip length $b_{\text {eff }}$ at the super-hydrophobic surface is defined as

$$
b_{\text {eff }}=\frac{\left\langle u_{z=0}\right\rangle}{\left\langle\left(\frac{\partial u}{\partial z}\right)_{z=0}\right\rangle},
$$

where $u$ denotes $x$-component of the velocity and $\langle\cdots\rangle$ means the average value in plane $x O y$.

The effective slip length can now be calculated by using the dual series technique suggested in recent work $[60,55]$. By employing a family of Fourier series solutions to equations (3), together with boundary conditions

$$
\begin{gathered}
\mathbf{u}(x, y, 0)=b(x, y) \cdot \frac{\partial \mathbf{u}}{\partial z}(x, y, 0), \quad \hat{\mathbf{z}} \cdot \mathbf{u}(x, y, 0)=0, \\
\mathbf{u}(x, y, H)=0, \quad \hat{\mathbf{z}} \cdot \mathbf{u}(x, y, H)=0,
\end{gathered}
$$

we obtain the dual series problem (in dimensionless form) for the longitudinal and transverse configurations

$\alpha_{0}\left(1+\frac{\beta}{h}\right)+\sum_{n=1}^{\infty} \alpha_{n}[1+\beta n V(n h)] \cos (n \xi)=\beta$,

$$
0<\xi \leqslant \pi \phi_{2}
$$

$\alpha_{0}+\sum_{n=1}^{\infty} \alpha_{n} \cos (n \xi)=0, \quad \pi \phi_{2}<\xi \leqslant \pi$,

where $\{\xi, h, \beta\}=(2 \pi / L) \cdot\{z, H, b\}$ and the function $V(x)$ is defined as

$$
V(x)=\operatorname{coth}(x)
$$

for a longitudinal flow and

$$
V(x)=2 \frac{\sinh (2 x)-2 x}{\cosh (2 x)-2 x^{2}-1}
$$

for a transverse flow. The effective slip length is then

$$
b_{\text {eff }}=\frac{L}{2 \pi} \frac{\alpha_{0}}{1-\alpha_{0} / h} .
$$

Following [60] we can now use the orthogonality of trigonometric sine and cosine functions to obtain a system of linear algebraic equations

$$
\sum_{n=0}^{\infty} A_{n m} \alpha_{n}=B_{m}
$$

that can be solved in respect to $\alpha_{n}$. Figure 8(a) shows the typical calculation results (the numerical example corresponds to $b / L=20$ and $\left.\phi_{2}=0.75\right)$ and demonstrates that the effective slip lengths increase with $H$ and saturate for a thick gap. This points to the fact that an effective boundary condition is not a characteristic of the liquid-solid interface alone, but depends on the flow configuration and interplay between the typical length scales, $L, H$ and $b$, of the problem. Next we discuss the asymptotic limits (of small and large gaps) of our semi-analytical solution. 
(a)

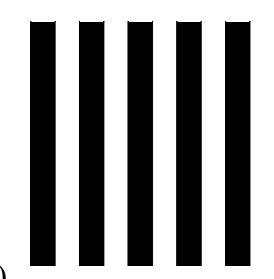

(b)

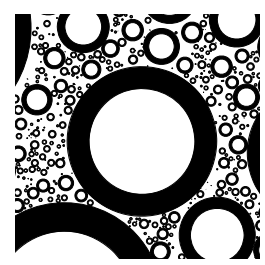

Figure 7. Special textures arising in the theory: (a) stripes, which attain the Wiener bounds of maximal and minimal effective slip, if oriented parallel or perpendicular to the pressure gradient, respectively; (b) the Hashin-Shtrikman fractal pattern of nested circles, which attains the maximal/minimal slip among all isotropic textures (patching should fill up the whole space, but their number is limited here for clarity); and (c) the Schulgasser and (d) chessboard textures, whose effective slip follows from the phase interchange theorem.

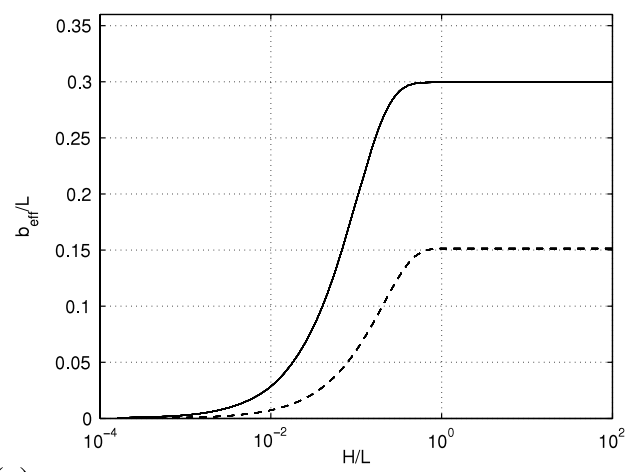

(a)

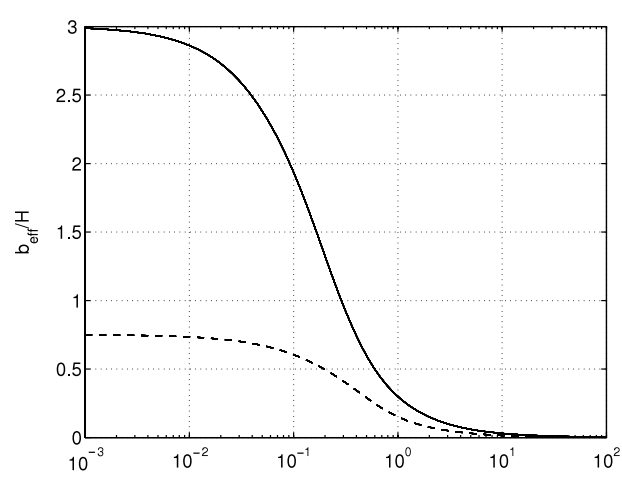

(b)

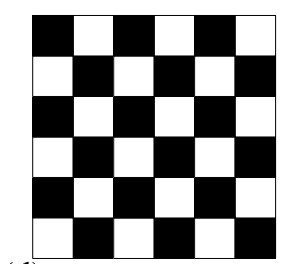

(d)

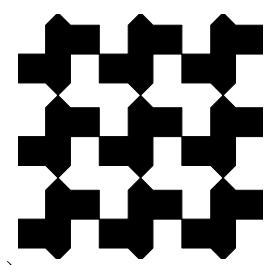

(c)

Figure 8. Eigenvalues $b_{\text {eff }}^{\|}$(solid curve) and $b_{\text {eff }}^{\perp}$ (dashed curve) of the slip-length tensor for stick-slip stripes of period $L$ with local slip length at the liquid-gas interface $b / L=20$ and slipping area fraction $\phi_{2}=0.75$, as a function of the thickness of the channel, $H$.

6.1.2. Thin channel. The Taylor expansion of $V(x)$ in the vicinity of $x=0$

$$
\begin{gathered}
\left.\operatorname{coth} x\right|_{x \rightarrow 0}=x^{-1}+O(x), \\
\left.2 \frac{\sinh (2 x)-2 x}{\cosh (2 x)-2 x^{2}-1}\right|_{x \rightarrow 0}=4 x^{-1}+O(x),
\end{gathered}
$$

allows us to find analytical expressions for $\alpha_{0}$ and $b_{\text {eff }}$ in the limit of $H \ll L$. By substituting them into (10) and (11), we get

$$
b_{\mathrm{eff}}^{\|} \simeq \frac{b H \phi_{2}}{H+b \phi_{1}}, \quad b_{\mathrm{eff}}^{\perp} \simeq \frac{b H \phi_{2}}{H+4 b \phi_{1}} .
$$

These expressions are independent of $L$, but depend on $H$, and suggest a way to distinguish between two separate cases.

If $b \ll H$ we obtain

$$
b_{\text {eff }}^{\perp} \simeq b_{\text {eff }}^{\|} \simeq b \phi_{2},
$$

so that despite the surface anisotropy we predict a simple surface-averaged effective slip. Although this limit is less important for pressure-driven microfluidics, it may have relevance for amplifying transport phenomena [65].

When $H \ll b$ we derive

$$
b_{\mathrm{eff}}^{\|} \simeq H \frac{\phi_{1}}{\phi_{2}}, \quad b_{\mathrm{eff}}^{\perp} \simeq \frac{1}{4} b_{\mathrm{eff}}^{\|}
$$

The above formula implies the effective slip length is generally four times as large for parallel versus perpendicular pressuredriven flow. Both asymptotic results, equations (19) and (20), are surprising taking into account that for anisotropic Stokes flow in a thick channel a factor of two is often expected, as reminiscent of results for striped pipes [66], sinusoidal grooves [16] and the classical result that a rod sediments twice as fast in creeping flow if aligned vertically rather than horizontally [67]. A very important conclusion from our analysis is that this standard scenario can significantly differ in a thin super-hydrophobic channel, giving a whole spectrum of possibilities, from isotropic to highly anisotropic flow, depending on the ratio $b / H$.

Note that in case of a thin channel the flow can be described by an expression of Darcy's law, which relates the depth-averaged fluid velocity to an average pressure gradient along the plates through the effective permeability of the channel. The permeability, $\mathbf{K}_{\text {eff }}$, is in turn expressed through the effective slip length $\mathbf{b}_{\text {eff }}$, and permeability and slip-length tensors are coaxial. Such an approach allows one to use the theory of transport in heterogeneous media [68], which provides exact results for an effective permeability over length scales much larger than the heterogeneity. This theory allows one to derive rigorous bounds on an effective slip length for arbitrary textures, given only the area fraction and local (any) slip lengths of the low-slip $\left(b_{1}\right)$ and high-slip $\left(b_{2}\right)$ regions [15, 69]. These bounds constrain the attainable effective slip and provide theoretical guidance for texture optimization, since they are attained only by certain special textures in the theory. In some regimes, the bounds are close enough to obviate the need for tedious calculations of flows over particular textures. In particular, by using the general result of the theory of bounds [15] one can easily derive 

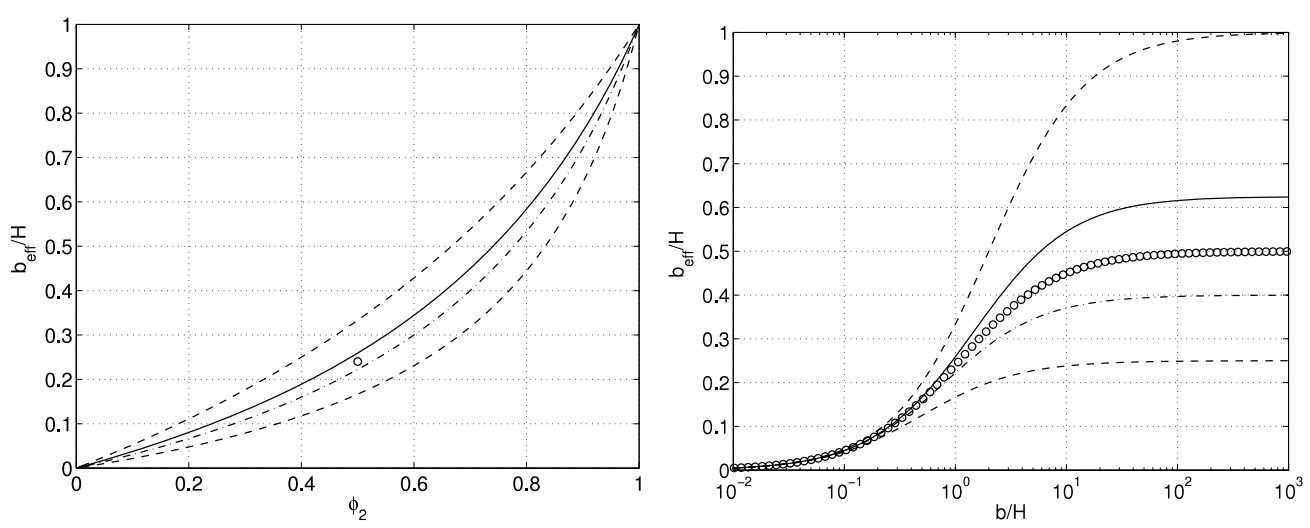

Figure 9. Effective slip length $b_{\text {eff }} / H$ versus $\phi_{2}$ (for $b / H=1$ ) (left) and $b / H$ (for $\phi_{2}=0.5$ ) (right) in the thin gap limit, $H \ll L$. SH surfaces are anisotropic stick-slip stripes attaining Wiener bounds (dashed curves), isotropic textures attaining Hashin-Shtrikman bounds (upper-solid, lower-dash-dotted curves) and satisfying the phase interchange theorem (circles).

equation (18) and its limits, as well as prove that for a thin channel longitudinal (transverse) stripes satisfy upper (lower) Wiener bounds, i.e. provide the largest (smallest) possible slip that can be achieved by any texture. We must stress that, according to the results [15], stripes need not necessarily be periodic.

Typical dependence of Wiener bounds for $b_{\text {eff }} / H$ on $\phi_{2}$ (at fixed $b / H$ ) and on $b / H$ (at fixed $\phi_{2}$ ) is shown in figure 9, which illustrates well that the key parameter determining effective slip in the thin channel is the area fraction of solid $\phi_{1}$ in contact with the liquid. If this is very small ( or $\phi_{2} \rightarrow 1$ ), for all textures the effective slip tends to a maximum value, $b_{\text {eff }} \rightarrow b$. We can also conclude that maximizing $b$ also plays a important role in achieving large effective slip.

6.1.3. Thick channel. In the opposite case of infinitely large thickness $(H \gg L)$ the dual series problems (10) and (11) reduce to those studied in recent work [55], due to $V(x \rightarrow \infty) \rightarrow 1$ and 2 for longitudinal and transverse stripes, respectively. Thus, the effective slip lengths in this case are [55]

$$
\begin{gathered}
b_{\text {eff }}^{\|} \simeq \frac{L}{\pi} \frac{\ln \left[\sec \left(\frac{\pi \phi_{2}}{2}\right)\right]}{1+\frac{L}{\pi b} \ln \left[\sec \left(\frac{\pi \phi_{2}}{2}\right)+\tan \left(\frac{\pi \phi_{2}}{2}\right)\right]}, \\
b_{\text {eff }}^{\perp} \simeq \frac{L}{2 \pi} \frac{\ln \left[\sec \left(\frac{\pi \phi_{2}}{2}\right)\right]}{1+\frac{L}{2 \pi b} \ln \left[\sec \left(\frac{\pi \phi_{2}}{2}\right)+\tan \left(\frac{\pi \phi_{2}}{2}\right)\right]} .
\end{gathered}
$$

The above results apply for a single surface, and are independent of $H$. However, these expressions for effective slip lengths depend strongly on the texture period $L$. When $b \ll L$ we again derive the area-averaged slip length, equation (19). When $b \gg L$, expressions (21) and (22) take the forms

$$
b_{\text {eff }}^{\perp} \simeq \frac{L}{2 \pi} \ln \left[\sec \left(\frac{\pi \phi_{2}}{2}\right)\right], \quad b_{\text {eff }}^{\|} \simeq 2 b_{\text {eff }}^{\perp},
$$

which coincide with the result obtained by Lauga and Stone [66] for the ideal slip $(b \rightarrow \infty)$ case. We stress that the commonly expected factor of two for the ratio of $b_{\text {eff }}^{\|}$and $b_{\text {eff }}^{\perp}$ is predicted only for very large $b / L$. In all other situations the anisotropy of the flow is smaller, and even disappears at moderate $b / L$.

\subsection{Isotropic surfaces}

As stressed above most solids are isotropic, i.e. without a preferred direction. Unfortunately, from the hydrodynamic point of view, this situation is more complicated than considered above. Below we discuss only some aspects of the hydrodynamic behavior in the thin and thick channel situations. For a thin channel, our arguments are based on the already mentioned theory of transport in heterogeneous media [68] and derived bounds on an effective slip length (effective slip length and permeability tensors are now becoming simply proportional to the unit tensor I) for arbitrary isotropic textures [15, 69]. For a thick channel, the only available arguments are based on the scaling theory and numerical calculations [54], which however does provide us with some guidance.

6.2.1. Thin channel. If the only knowledge about the two-phase isotropic texture is $\phi_{1}$ and $\phi_{2}$, then the HashinShtrikman (HS) bounds apply for the effective permeability, by giving the corresponding upper and lower HS bounds for the effective slip length $[15,69]$. These bounds can be attained by the special HS fractal pattern sketched in figure 7(b). For one bound, space is filled by discs of all sizes, each containing a circular core of one component and a thick ring of the other (with proportions set by the concentration), and switching the components gives the other bound. Fractal geometry is not necessary, however, since periodic honeycomb-like structures can also attain these bounds. The general solution [15, 69] allows one to deduce consequential analytical results for the asymmetric case considered in this paper. The upper (HS) bound can then be presented as

$$
b_{\text {eff }}=\frac{b H \phi_{2}(2 H+5 b)}{H(2 H+5 b)+b \phi_{1}(5 H+b)},
$$


and the lower (HS) bound reads

$$
b_{\text {eff }}=\frac{2 b H \phi_{2}}{2 H+5 b \phi_{1}} .
$$

At small $b / H$ we get equation (19), and at large $b / H$ these give for upper and lower bounds

$$
b_{\text {eff }}=\frac{5 H \phi_{2}}{8 \phi_{1}}, \quad \text { and } \quad b_{\text {eff }}=\frac{2 H \phi_{2}}{5 \phi_{1}},
$$

correspondingly.

Finally, phase interchange results [15] can be applied for some specific patterns (figures 7 (c) and (d)). The phase interchange theorem states that the effective permeability $\mathbf{K}_{\text {eff }}\left(b_{1}, b_{2}\right)$ of the medium, when rotated by $\pi / 2$, is related to the effective permeability of the medium obtained by interchanging phases 1 and 2, namely $\mathbf{K}_{\mathrm{eff}}\left(b_{2}, b_{1}\right)$ :

$$
\left[\mathbf{R} \cdot \mathbf{K}_{\mathrm{eff}}\left(b_{1}, b_{2}\right) \cdot \mathbf{R}^{\mathrm{t}}\right] \cdot \mathbf{K}_{\mathrm{eff}}\left(b_{2}, b_{1}\right)=K_{1} K_{2} \mathbf{I}
$$

where $b_{1,2}$ are the local slip lengths for each medium, $\mathbf{R}$ is the rotation tensor and $\mathbf{R}^{t}$ is its transpose. In the particular case of a medium which is invariant by a $\pi / 2$ rotation followed by a phase interchange, the classical result follows:

$$
K_{\text {eff }}=\sqrt{K_{1} K_{2}}
$$

Obviously, $\phi_{1}=\phi_{2}=0.5$ for such a medium so that

$$
b_{\mathrm{eff}}=\frac{3 H}{4-\sqrt{1+\frac{3 b}{H+b}}}-H .
$$

At $b / H \ll 1$ we again derive equation (19), indicating that at this limit all textures show a kind of universal behavior and the effective slip coincides with the average. This suggests that the effective slip is controlled by the smallest scale of the problem $[9,55]$, so that at this limit $b_{\text {eff }}$ is no longer dependent on $H$, being proportional to $b$ only. If $b / H \gg 1$ we simply get

$$
b_{\text {eff }}=\frac{H}{2}
$$

again suggesting a kind of universality, i.e. similar to anisotropic stripes (cf equation (20)), in this limit $b_{\text {eff }} / H$ for all isotropic textures are likely to scale $\propto \phi_{2} / \phi_{1}$.

The results for these special textures are included in figure 9, which shows that HS bounds are relatively close and confined between Wiener ones. For completeness, we give in table 1 a summary of the main expressions for an effective slip in a thin channel.

6.2.2. Thick channel. For this situation the exact solution has not been found so far. Nevertheless, some simple scaling expressions have been proposed for a geometry of pillars $[9,54]$, predicting $b_{\text {eff }} \propto L /\left(\pi \sqrt{\phi_{1}}\right.$ ) (cf scaling results for stripes $\left.b_{\text {eff }} \propto L / \ln \left(1 / \phi_{1}\right)\right)$. This simple result still deserves some analytical justification, which has not been performed up to now.
Table 1. The effective slip length $b_{\text {eff }}$ for different textures (shown in figure 7) in a thin gap limit $(H \ll L)$.

\begin{tabular}{ll}
\hline Texture & $b_{\text {eff }}$ \\
\hline $\begin{array}{l}\text { Wiener upper bound } \\
\text { (longitudinal stripes) }\end{array}$ & $\frac{b H \phi_{2}}{H+b \phi_{1}}$ \\
$\begin{array}{l}\text { Wiener lower bound (transverse } \\
\text { stripes) }\end{array}$ & $\frac{b H \phi_{2}}{H+4 b \phi_{1}}$ \\
$\begin{array}{l}\text { HS upper bound (HS fractal, } \\
\text { honeycomb-like texture) }\end{array}$ & $\frac{b H \phi_{2}(2 H+5 b)}{H(2 H+5 b)+b \phi_{1}(5 H+b)}$ \\
$\begin{array}{l}\text { HS lower bound (HS fractal, } \\
\text { honeycomb-like texture) }\end{array}$ & $\frac{2 b H \phi_{2}}{2 H+5 b \phi_{1}}$ \\
$\begin{array}{l}\text { Phase interchange patterns } \\
\text { (Schulgasser texture, family of } \\
\text { chessboards) }\end{array}$ & $\frac{3 H}{4-\sqrt{1+3 b /(H+b)}}-H$ \\
\hline
\end{tabular}

\section{Other special properties of super-hydrophobic surfaces}

As we see above, hydrophobic Cassie materials generate large and anisotropic effective slippage compared to simple, smooth channels, an ideal situation for various potential applications. A straightforward application of super-hydrophobic slip would be the great reduction of the viscous drag of thin microchannels (enhanced forward flow), and some useful examples can be found in [14]. Below we illustrate the potential of superhydrophobic surfaces and the possibilities of the effective slip approach by discussing a couple of other applications. Namely, we show that optimized super-hydrophobic textures may be successfully used in a passive microfluidic mixing and for reduction of the hydrodynamic drag force.

\subsection{Transverse flow}

The effective hydrodynamic slip [3, 14, 16] of anisotropic textured surfaces is generally tensorial, which is due to secondary flows transverse to the direction of the applied pressure gradient. In the case of grooved no-slip surfaces (Wenzel state), such a flow has been analyzed for small height variations [70] and thick channels [71], and herringbone patterns have been designed to achieve passive chaotic mixing during pressure-driven flow through a microchannel [64, 72]. In principle, similar effects may be generated by a superhydrophobic Cassie surface. To explore this possibility and to illustrate the use of slip tensors we consider now a velocity field in a channel with the focus on transverse flow optimization, which is necessary for mixing in a microfluidic channel.

By solving the Stokes equations (3) for a geometry of super-hydrophobic stripes with an effective boundary condition at $z=0$ :

$$
\begin{aligned}
\left(\begin{array}{c}
\left\langle u_{x}\right\rangle \\
\left\langle u_{y}\right\rangle
\end{array}\right) & \\
= & \left(\begin{array}{cc}
b_{\mathrm{eff}}^{\|} \cos ^{2} \theta+b_{\mathrm{eff}}^{\perp} \sin ^{2} \theta & \left(b_{\mathrm{eff}}^{\|}-b_{\mathrm{eff}}^{\perp}\right) \sin \theta \cos \theta \\
\left(b_{\mathrm{eff}}^{\|}-b_{\mathrm{eff}}^{\perp}\right) \sin \theta \cos \theta & b_{\mathrm{eff}}^{\|} \sin ^{2} \theta+b_{\mathrm{eff}}^{\perp} \cos ^{2} \theta
\end{array}\right) \\
& \cdot\left(\begin{array}{c}
\left\langle\partial_{z} u_{x}\right\rangle \\
\left\langle\partial_{z} u_{y}\right\rangle
\end{array}\right)
\end{aligned}
$$



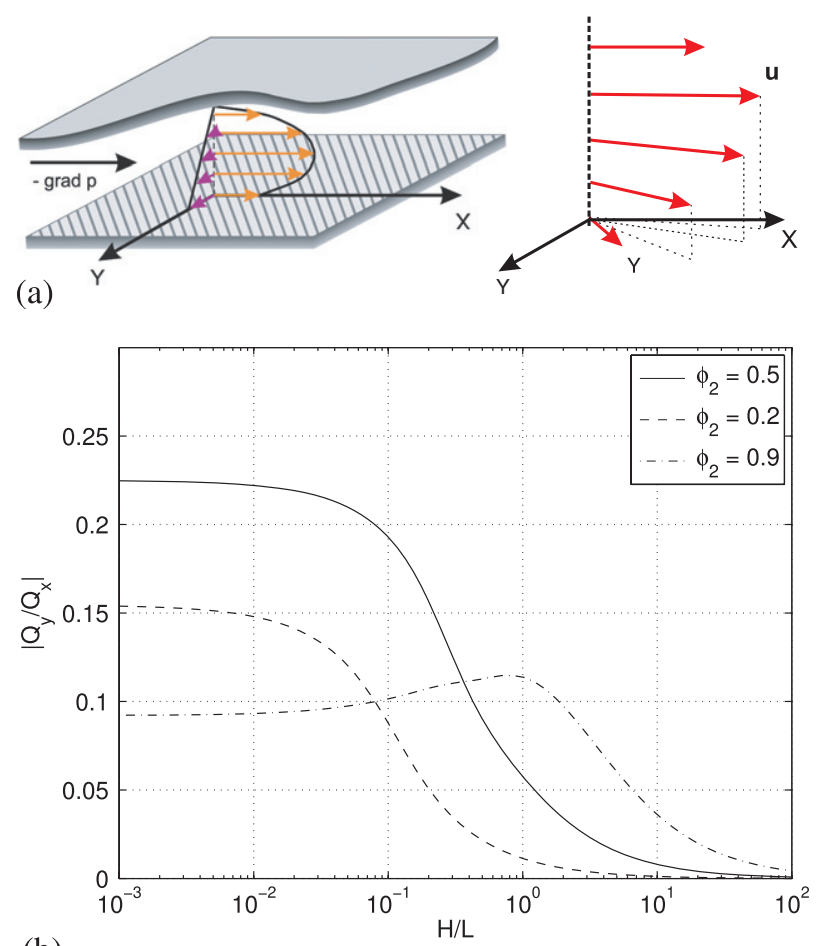

(b)

Figure 10. (a) Scheme of transverse flow generation. (b) The ratio of flow vector $\mathbf{Q}$ components (maximized over $\theta$ ) as a function of channel thickness for local slip $b / L=1000$ and slip fraction $\phi_{2}=0.5$ (solid line), 0.2 (dashed) and 0.9 (dash-dotted).

we obtain components of the effective velocity

$$
\begin{gathered}
\left\langle u_{x}\right\rangle=-\frac{\sigma z^{2}}{2 \eta}+\frac{\sigma H z}{2 \eta}+C_{x}\left(1-\frac{z}{H}\right), \\
\left\langle u_{y}\right\rangle=C_{y}\left(1-\frac{z}{H}\right)
\end{gathered}
$$

with

$$
\begin{gathered}
C_{x}=\frac{\sigma H^{2}}{2 \eta} \frac{H b_{\mathrm{eff}}^{\|} \cos ^{2} \theta+H b_{\mathrm{eff}}^{\perp} \sin ^{2} \theta+b_{\mathrm{eff}}^{\|} b_{\mathrm{eff}}^{\perp}}{\left(H+b_{\mathrm{eff}}^{\|}\right)\left(H+b_{\mathrm{eff}}^{\perp}\right)}, \\
C_{y}=\frac{\sigma H^{3}}{2 \eta} \frac{\left(b_{\mathrm{eff}}^{\|}-b_{\mathrm{eff}}^{\perp}\right) \sin \theta \cos \theta}{\left(H+b_{\mathrm{eff}}^{\|}\right)\left(H+b_{\mathrm{eff}}^{\perp}\right)} .
\end{gathered}
$$

Note that our results show that the effective velocity profile is 'twisted' close to the super-hydrophobic wall (see figure 10(a)). In other words, the transverse flow due to surface anisotropy is generated only in the vicinity of the wall and disappears far from it, which has already been observed in experiment [73].

To evaluate the transverse flow we first integrate the velocity profile across the channel to obtain the flow vector:

$$
\mathbf{Q}=\int_{0}^{H}\langle\mathbf{u}(z)\rangle \mathrm{d} z
$$

with the components (according to (29))

$Q_{x}=\frac{\sigma}{\eta} \frac{H^{3}}{12}\left[1+3 \frac{\left(H b_{\mathrm{eff}}^{\|} \cos ^{2} \theta+H b_{\mathrm{eff}}^{\perp} \sin ^{2} \theta+b_{\mathrm{eff}}^{\|} b_{\mathrm{eff}}^{\perp}\right)}{\left(H+b_{\mathrm{eff}}^{\|}\right)\left(H+b_{\mathrm{eff}}^{\perp}\right)}\right]$,

$$
Q_{y}=\frac{\sigma}{\eta} \frac{H^{4}}{4} \frac{\left(b_{\mathrm{eff}}^{\|}-b_{\mathrm{eff}}^{\perp}\right) \sin \theta \cos \theta}{\left(H+b_{\mathrm{eff}}^{\|}\right)\left(H+b_{\mathrm{eff}}^{\perp}\right)} .
$$

Next we consider the fraction $\left|Q_{y} / Q_{x}\right|$. Our aim is to optimize the texture, channel thickness and the angle $\theta$ between the directions of stripes and the pressure gradient, so that $\left|Q_{y} / Q_{x}\right|$ is maximum, providing the best transverse flow.

The maximization in respect to $\theta$ indicates that the optimal angle is

$$
\theta_{\max }= \pm \arctan \left[\frac{\left(1+4 b_{\mathrm{eff}}^{\|} / H\right)\left(1+b_{\mathrm{eff}}^{\perp} / H\right)}{\left(1+b_{\mathrm{eff}}^{\|} / H\right)\left(1+4 b_{\mathrm{eff}}^{\perp} / H\right)}\right]^{1 / 2} .
$$

The value of the maximum is

$$
\left|\frac{Q_{y}}{Q_{x}}\right|=\frac{1}{2}\left(\tan \theta_{\max }-\frac{1}{\tan \theta_{\max }}\right) .
$$

We conclude, therefore, that since $H$ is fixed, the maximal $\left|Q_{y} / Q_{x}\right|$ corresponds to the largest physically possible $b$, i.e. perfect slip at the gas sectors.

To optimize the fraction of the slipping area $\phi_{2}$ we should now exploit the results for effective slip lengths $b_{\text {eff }}^{\|, \perp}$ obtained above. Figure 10(b) shows the computed value of $\left|Q_{y} / Q_{x}\right|$ versus $H / L$ for several $\phi_{2}$. The calculations are made using the value of $\theta$ defined by equation (33). The data suggest that the effect of $\phi_{2}$ on a transverse flow depends on the thickness of the channel. For a thick gap the increase in gas fraction $\phi_{2}$ augments a transverse flow. This result has a simple explanation. For an infinite channel $b_{\text {eff }}^{\|, \perp} / H \ll 1$ (see figure 9(b)), which gives

$$
\left|\frac{Q_{y}}{Q_{x}}\right|_{H \rightarrow \infty} \simeq \frac{3}{2} \frac{\Delta b_{\mathrm{eff}}}{H}
$$

i.e. in a thick channel the amplitude of a transverse flow is controlled by the difference between the eigenvalues of the effective slip tensor $\Delta b_{\text {eff }}=b_{\text {eff }}^{\|}-b_{\text {eff }}^{\perp}$ which increases with $\phi_{2}$ as follows from the above analysis. We stress, however, that since $\left|Q_{y} / Q_{x}\right| \propto H^{-1}$, the mixing in a thick superhydrophobic channel would be not very efficient. A more appropriate situation corresponds to a thin channel, as is well illustrated in figure 10(b). We see that the largest transverse flow can be generated at intermediate values of $\phi_{2}$. The limit of a thin channel has recently been studied in detail by using a general theory of mathematical bounds [68] and the optimum value of $\phi_{2}=0.5$ corresponding a numerical example in figure 10(b) has been rigorously derived [74].

An important conclusion from our analysis is that the surface textures which optimize transverse flow can differ significantly from those optimizing effective (forward) slip. It is well known, and we additionally demonstrated above, that the effective slip of a super-hydrophobic surface is maximized by reducing the solid-liquid area fraction $\phi_{1}$. In contrast, we have shown that transverse flow in super-hydrophobic channels is maximized by stripes with a rather large solid fraction, $\phi_{1}=0.5$, where the effective slip is relatively small. 


\subsection{Hydrodynamic interactions}

As a consequence of the superlubrication potential, a hydrophobic texture could significantly modify squeeze film drainage between surfaces. It is of obvious practical interest to minimize the hydrodynamic resistance, $F$, to the approach of surfaces.

For a Reynolds problem, where a disc of radius $R$ is driven towards (in our case) a super-hydrophobic plane with a velocity $U$ (see figure 11), we should solve equations (3) by applying the effective tensorial boundary condition, equation (4), at the super-hydrophobic wall. This allows us to derive a general expression for hydrodynamic pressure satisfying the condition $p=p_{0}$ at the edge of the disc [75]

$$
p=p_{0}+\frac{U}{2} \frac{\left(R^{2}-r^{2}\right)}{\left(C_{x}+C_{y}\right)}, \quad r^{2}=x^{2}+y^{2},
$$

where

$$
C_{x}=\frac{H^{3}}{12 \eta} \frac{H+4 b_{\mathrm{eff}}^{\|}}{H+b_{\mathrm{eff}}^{\|}}, \quad C_{y}=\frac{H^{3}}{12 \eta} \frac{H+4 b_{\mathrm{eff}}^{\perp}}{H+b_{\mathrm{eff}}^{\perp}} .
$$

The drag force may then be evaluated as the integral over the disc's surface and reads [75]

$$
F=\frac{3}{2} \frac{\pi \eta U R^{4}}{H^{3}} f_{\mathrm{eff}}^{*}=F_{\mathrm{R}} f_{\mathrm{eff}}^{*},
$$

where $F_{\mathrm{R}}$ represents the classical solution of the creeping flow equations of the Reynolds lubrication theory [76] and the correction for an effective slip is

$$
f_{\mathrm{eff}}^{*}=\frac{F}{F_{\mathrm{R}}}=2\left[\frac{H+4 b_{\mathrm{eff}}^{\|}(H)}{H+b_{\mathrm{eff}}^{\|}(H)}+\frac{H+4 b_{\mathrm{eff}}^{\perp}(H)}{H+b_{\mathrm{eff}}^{\perp}(H)}\right]^{-1} .
$$

Thus the effective correction for a super-hydrophobic slip is the harmonic mean of the corrections expressed through effective slip lengths in the two principal directions:

$$
f_{\mathrm{eff}}^{*}=2\left(\frac{1}{f_{\mathrm{eff}}^{*\|\|}}+\frac{1}{f_{\mathrm{eff}}^{*, \perp}}\right)^{-1}
$$

In the case of isotropic textures, all directions are equivalent with $b_{\text {eff }}^{\|}=b_{\text {eff }}^{\perp}=b_{\text {eff }}$, so we get

$$
f_{\mathrm{eff}}^{*}=\frac{F}{F_{\mathrm{R}}}=\frac{H+b_{\mathrm{eff}}(H)}{H+4 b_{\mathrm{eff}}(H)} .
$$

Obviously, the case $b_{\text {eff }}^{\|}=b_{\text {eff }}^{\perp}=0$ corresponds to $f_{\text {eff }}^{*}=1$ and gives the Reynolds formula.

The expression for $f_{\text {eff }}^{*}$ is very general and relates it to the effective slip length of the super-hydrophobic wall and the gap. In order to quantify the reduction of a drag force due to a presence of a super-hydrophobic wall, this expression can be used for all anisotropic and isotropic textures where analytical or numerical expressions for $b_{\text {eff }}^{\|, \perp}$ have been obtained.

An important consequence of equation (39) is that to reduce a drag force we need to maximize the ratio $b_{\text {eff }} / H$, but not the absolute values of effective slip itself. This is illustrated
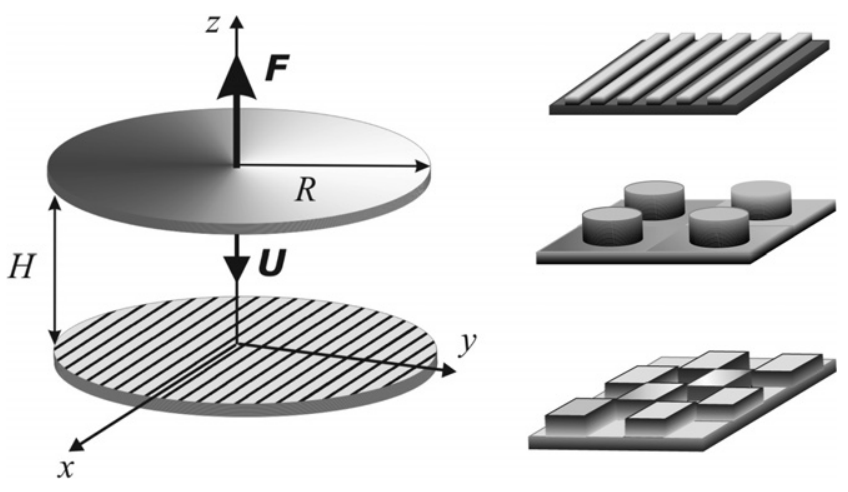

Figure 11. (Left) Sketch of a hydrophilic disc approaching a super-hydrophobic striped disc. (Right) Examples of anisotropic (grooves) and isotropic (pillars, chessboard) textures.

Table 2. Asymptotic expansions for the force correction factor $f_{\mathrm{eff}}^{*}$ in the case of a striped surface.

\begin{tabular}{ll}
\hline Limiting case & $f_{\text {eff }}^{*}$ \\
\hline$H \gg \max \{L, b\}$ & $1-\frac{3\left(b_{\mathrm{eff}}^{\|}+b_{\mathrm{eff}}^{\perp}\right)}{2 H}$ \\
$L \ll H \ll b$ & $\frac{1}{4}+\frac{9}{32} \frac{\pi H}{L \ln \left(\sec \left(\pi \phi_{2} / 2\right)\right)}$ \\
$b \ll H \ll L$ & $1-\frac{3 b \phi_{2}}{H}$ \\
$H \ll \min \{L, b\}$ & $\frac{2\left(4-3 \phi_{2}\right)}{8+9 \phi_{2}-9 \phi_{2}^{2}}$ \\
\hline
\end{tabular}

Table 3. Correction factor $f_{\text {eff }}^{*}$ for some specific isotropic patterns in the thin gap limit.

\begin{tabular}{lcl}
\hline Pattern & $b \ll H \ll L$ & $H \ll \min \{L, b\}$ \\
\hline Hashin-Shtrikman upper bound & $1-\frac{3 b \phi_{2}}{H}$ & $\frac{5-3 \phi_{2}}{5+3 \phi_{2}}$ \\
Hashin-Shtrikman lower bound & $1-\frac{3 b \phi_{2}}{H}$ & $\frac{8-3 \phi_{2}}{4\left(2+3 \phi_{2}\right)}$ \\
Phase interchange textures & $1-\frac{3 b}{2 H}$ & $\frac{1}{2}$ \\
\hline
\end{tabular}

in figure 12(a), where values presented in figure $8(\mathrm{~b})$ were used to compute the correction for effective slip, $f_{\text {eff }}^{*}$, as a function of the gap. At small $H / L$ our calculations reproduce the asymptotic values predicted by equations (20). They vanish, however, at large distances, where $b_{\mathrm{eff}}^{\|, \perp} / H$ becomes negligibly small. The useful analytical expressions for $f_{\text {eff }}^{*}$ corresponding to a configuration of stripes are presented in table 2. Similar estimates for the most important situation of a thin gap can be made for some isotropic textures, and we include these results in table 3 .

The results presented in tables 2 and 3 suggest that the key parameter determining reduction of drag is the area fraction of gas, $\phi_{2}$, in contact with the liquid. This is illustrated in figure 12(b), where (using a relatively large $b / H$ ) HS bounds for $f_{\text {eff }}^{*}$ are plotted versus $\phi_{2}$. If this is very small (or $\phi_{1} \rightarrow 1$ ) for all textures, the correction for slip tends to its absolute maximum, $f_{\text {eff }}^{*} \rightarrow 1$. In the most interesting limit, $\phi_{2} \rightarrow 1$, we can achieve the minimum possible value of correction for 


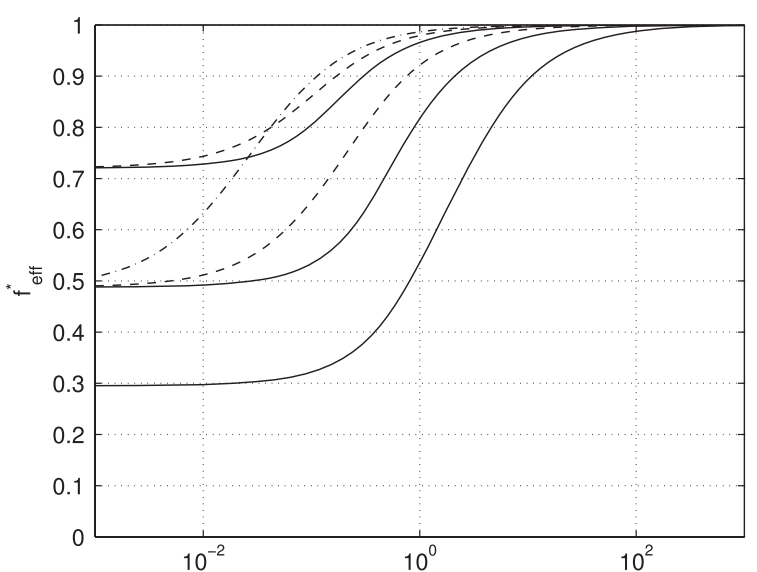

(a)

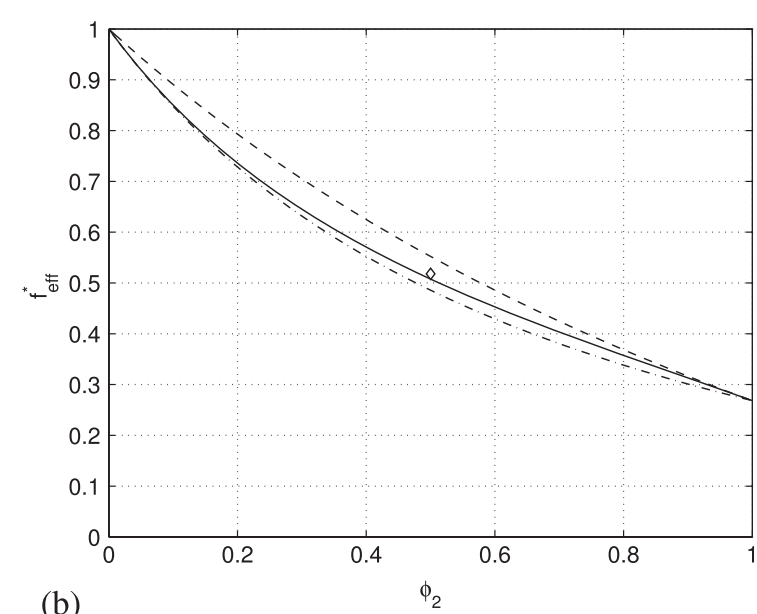

(b)

Figure 12. (a) The correction factor $f_{\text {eff }}^{*}=F / F_{\mathrm{R}}$ for the hydrodynamic resistance force exerted on a disc interacting with super-hydrophobic stripes versus dimensionless gap width $H / L$. Solid curves correspond to local slip length $b / L=10$ (from top to bottom $\phi_{2}=0.2$, 0.5 and 0.9 ), dashed curves to $b / L=0.1$ (from top to bottom $\phi_{2}=0.2$ and 0.5 ), dash-dotted curve to $b / L=0.01$ and $\phi_{2}=0.5$. (b) The plot of $f_{\text {eff }}^{*}$ versus $\phi_{2}$ for a thin gap $(H \ll L)$ and several super-hydrophobic patterns: anisotropic stripes (solid line), isotropic textures attaining HS bounds (dashed and dash-dotted lines) and isotropic Schulgasser structure (diamond), all with local slip $b / H=10$.

effective slip, $f_{\text {eff }}^{*} \rightarrow 1 / 4$, provided $b / H$ is large enough. We also stress that the results for stripes are confined between HS bounds for $f_{\mathrm{eff}}^{*}$. In other words, isotropic textures might be the best candidates for a reduction of the drag force.

\section{Electro-osmotic flow over super-hydrophobic surfaces}

Electro-osmosis, i.e. flow generation by an electric field, represents an example of interfacially driven flows [65], which are currently intensively used in microfluidics. It may be enhanced by surface slippage, even for nanometric slip length. The reason for this amplification is that the electric double layer (EDL), characterized by the Debye screening length $\lambda_{\mathrm{D}}=\kappa^{-1}$ defines an additional length scale of the problem comparable to $b$. According to the classical formula $[77,78]$

$$
u=-\frac{q_{0} E_{0}}{\eta \kappa}(1+b \kappa)
$$

where $u$ is the electro-osmotic velocity (outside of the double layer), $q_{0}$ is the surface charge density, and $E_{0}$ is the tangential electric field. Therefore, the flow can potentially be enhanced for a thin, compared to $b$, EDL, i.e. when $\kappa b \gg 1$ (see figure 13).

For this reason, it is attractive to consider electroosmotic flow over super-hydrophobic surfaces, whose texture can significantly amplify hydrodynamic slip. Equation (42) suggests that a massive amplification of electro-osmotic flow can be achieved over super-hydrophobic surfaces. However, the controlled generation of such flows is by no means obvious, since both the slip length and charge distribution are inhomogeneous and anisotropic. Despite its fundamental and practical significance, electro-osmotic flow over superhydrophobic surfaces has received little attention.

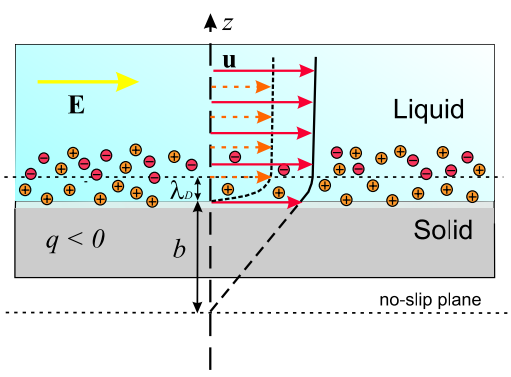

Figure 13. Sketch of the influence of slippage on the electro-osmotic flow.

Recently, such a flow has been investigated past an inhomogeneously charged slipping surface in the case of a thick channel $(H \gg L)$ and perfect $\operatorname{slip}(b \rightarrow \infty)$ at the gas sectors. The summary of results for the case of stripes $[79,80]$ is given in table 4 and we comment below on some useful limiting situations. Note that here we have corrected erroneous expressions for a transverse electro-osmotic velocity given in [80]. The details of our analysis will be described elsewhere. For a thin EDL $(\kappa L \gg 1)$, no flow enhancement is predicted in the case of an uncharged gas interface [79], which has been confirmed by molecular dynamic simulations [81] and later analysis [80]. For an uncharged gas interface with thick EDL $(\kappa L \ll 1)$, the results are qualitatively different and the electro-osmotic flow is directly set by the hydrodynamic effective slip-length tensor [80]. However, since $\kappa L$ is small and since the electro-osmotic velocity becomes proportional to $\phi_{1}$, electro-osmotic flow becomes suppressed despite a large effective slip (see figure 14(a)). One main conclusion is that a charged gas interface is required to achieve an enhancement of electro-osmotic flow. For a uniformly charged anisotropic super-hydrophobic surface the electro-osmotic mobility is isotropic and can exhibit a large enhancement from effective 


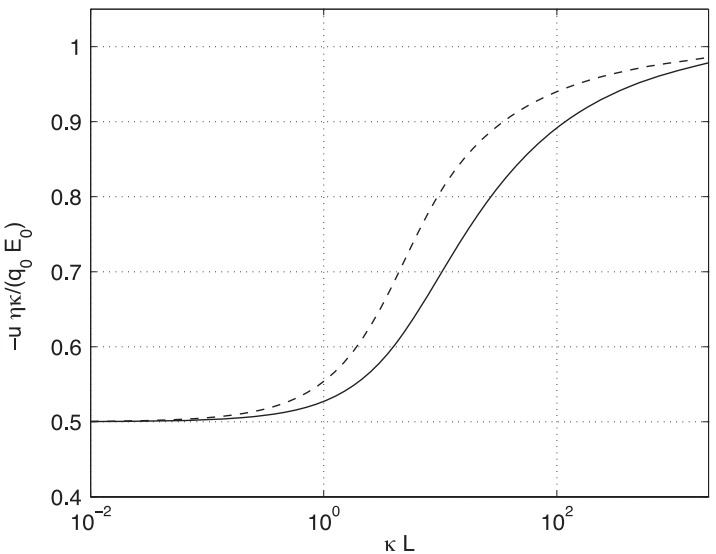

(a)

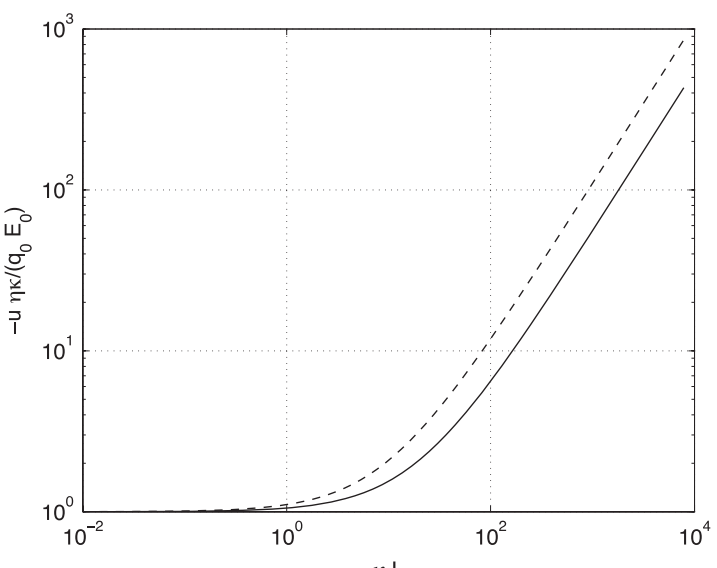

(b)

Figure 14. The electro-osmotic slip velocities for eigendirections of super-hydrophobic stripes $\left(\phi_{2}=0.5\right.$ and $\left.b / L \gg 1\right)$ for (a) uncharged gas sectors $q_{1}=q_{0}, q_{2}=0$ and (b) a surface of constant charge $q_{1}=q_{2}=q_{0}$. Solid curves correspond to transverse, and dashed to longitudinal EO flow.

Table 4. Electro-osmotic slip past super-hydrophobic stripes.

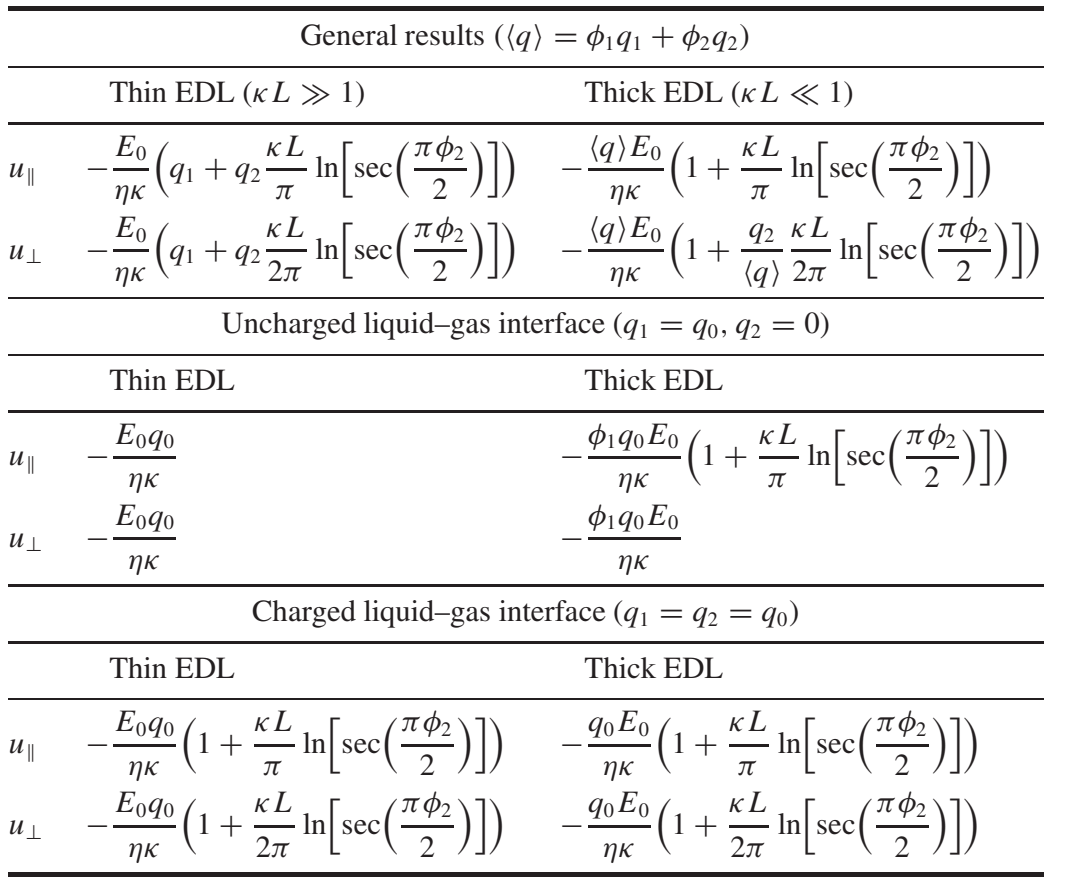

hydrodynamic slip, possibly by an order of magnitude (see figure 14(b)).

In the future, we suggest as a fruitful direction to consider electro-osmotic flow in a thin gap and assuming a partial slip at the gas sectors. If similar relations hold for thin channels, then our results for the effective hydrodynamic slip suggest that transverse electrokinetic phenomena could be greatly amplified by using striped super-hydrophobic surfaces.

\section{Conclusion}

With recent progress in micro- and nanofluidics a new interest has arisen in determining the forms of the hydrodynamic boundary conditions $[3,14,16]$. In particular, advances in lithography in patterning substrates have raised several questions in the modeling of the motion of liquids over these surfaces and led to the concept of the effective tensorial slip. These effective conditions capture the complicated effects of surface anisotropy and can be used to quantify the flow over complex textures without the tedium of enforcing real inhomogeneous boundary conditions.

This work has discussed the issue of boundary conditions at smooth hydrophobic and rough hydrophilic surfaces and has then given the especial emphasis to the derivation of effective boundary conditions for a flow past hydrophobic solid surfaces with special textures that can exhibit greatly enhanced ('super') properties, compared to analogous flat or slightly disordered surfaces [5]. After a decade of intense research there have been hundreds of materials developed and some macroscopic 
applications (such as self-cleaning, for example), but there are still no real small scale applications (for example, micro- and nanofluidics).

We have derived accurate formulas describing effective boundary conditions for pressure-driven flow past superhydrophobic textures of special interest (such as stripes, fractal patterns of nested circles, chessboards, etc). We have analyzed both thin (compared to texture characteristic length) and thick channel situations, and in some cases (e.g. periodic stripes) have obtained exact solutions valid for an arbitrary thickness of the channel. The predicted large effective slip of superhydrophobic surfaces compared to simple, smooth channels can greatly lower the viscous drag of thin microchannels.

The power of the tensor formalism and the concept of effective slippage was then demonstrated by exact solutions for two other potential applications: optimization of the transverse flow and analytical results for the hydrodynamic resistance to the approach of two surfaces. These examples demonstrate that properly designed super-hydrophobic surfaces could generate a very strong transverse flow and significantly reduce the so-called 'viscous adhesion'. A striking conclusion from our analysis is that the surface textures which optimize transverse flow or thin film drainage can differ significantly from those optimizing the effective (forward) slip. Finally, we have discussed how super-hydrophobic surfaces could amplify electrokinetic pumping in microfluidic devices.

A combination of wetting and roughness provides many new and very special hydrodynamic properties of surfaces that could be explored with the formalism discussed here. We hope our analysis will allow the local slip tensors to be determined by global measurements, such as the permeability of a textured channel as a function of the surface orientations or the hydrodynamic force exerted on a body approaching superhydrophobic plates. They may also guide the design of superhydrophobic surfaces for microfluidic lab-on-a-chip and other applications.

\section{Acknowledgments}

This research was supported by the DFG through its priority program 'Micro- and Nanofluidics' and by the RAS through its priority program 'Assembly and Investigation of Macromolecular Structures of New Generations'.

\section{References}

[1] Batchelor G K 2000 An Introduction to Fluid Dynamics (Cambridge: Cambridge University Press)

[2] Squires T M and Quake S R 2005 Rev. Mod. Phys. 77977

[3] Stone H A, Stroock A D and Ajdari A 2004 Annu. Rev. Fluid Mech. 36381

[4] Vinogradova O I 1999 Int. J. Miner. Proc. 5631

[5] Quere D 2005 Rep. Prog. Phys. 682495

[6] Vinogradova O I 1995 Langmuir 112213

[7] Vinogradova O I 1995 J. Colloid Interface Sci. 169306

[8] Lauga E, Brenner M P and Stone H A 2007 Handbook of Experimental Fluid Dynamics (New York: Springer) chapter 19, pp 1219-40

[9] Bocquet L and Barrat J L 2007 Soft Matter 3685

[10] Huang D, Sendner C, Horinek D, Netz R and Bocquet L 2008 Phys. Rev. Lett. 101226101
[11] Sendner C, Horinek D, Bocquet L and Netz R 2009 Langmuir 2510768

[12] Andrienko D, Dünweg B and Vinogradova O I 2003 J. Chem. Phys. 11913106

[13] Dammler S M and Lohse D 2006 Phys. Rev. Lett. 96206101

[14] Bazant M Z and Vinogradova O I 2008 J. Fluid Mech. 613125

[15] Feuillebois F, Bazant M Z and Vinogradova O I 2009 Phys. Rev. Lett. 102026001

[16] Kamrin K, Bazant M and Stone H A 2010 J. Fluid Mech. 658409

[17] Quere D 2008 Annu. Rev. Mater. Res. 3871

[18] Chan D and Horn R 1985 J. Chem. Phys. 835311

[19] Cottin-Bizonne C, Cross B, Steinberger A and Charlaix E 2005 Phys. Rev. Lett. 94056102

[20] Horn R G, Vinogradova O I, Mackay M E and Phan-Thien N 2000 J. Chem. Phys. 1126424

[21] Vinogradova O I and Yakubov G E 2003 Langmuir 191227

[22] Vinogradova O I 1996 Langmuir 125963

[23] Pit R, Hervet H and Leger L 2000 Phys. Rev. Lett. 85980

[24] Tretheway D C and Meinhart C D 2002 Phys. Fluids 14 L9

[25] Joseph P and Tabeling P 2005 Phys. Rev. E 71 035303(R)

[26] Huang P, Guasto J and Breuer K 2006 J. Fluid Mech. 566447

[27] Zettner C M and Yoda M 2003 Exp. Fluids 34115

[28] Li H, Sadr R and Yoda M 2006 Exp. Fluids 41185

[29] Vinogradova O I, Koynov K, Best A and Feuillebois F 2009 Phys. Rev. Lett. 102118302

[30] Lumma D, Best A, Gansen A, Feuillebois F, Rädler J O and Vinogradova O I 2003 Phys. Rev. E 67056313

[31] Joly L, Ybert C and Bocquet L 2006 Phys. Rev. Lett. 96046101

[32] Yordanov S, Best A, Butt H J and Koynov K 2009 Opt. Express 1721150

[33] Rothstein J P 2010 Annu. Rev. Fluid Mech. 4289

[34] Neto C, Evans D, Bonaccurso E, Butt H J and Craig V J 2005 Rep. Prog. Phys. 682859

[35] Bocquet L and Charlaix E 2010 Chem. Soc. Rev. 391073

[36] Kunert C, Harting J and Vinogradova O I 2010 Phys. Rev. Lett. 105016001

[37] Thompson P A and Troian S M 1997 Nature 389360

[38] Harting J, Kunert C and Herrmann H 2006 Europhys. Lett. 75328

[39] Vinogradova O I and Yakubov G E 2006 Phys. Rev. E 73 045302(R)

[40] Honig C D F and Ducker W A 2007 Phys. Rev. Lett. 98028305

[41] Bouzigues C I, Tabeling P and Bocquet L 2008 Phys. Rev. Lett. 101114503

[42] Maali A, Hurth C, Cohen-Bouhacina T, Gouturier G and Aime J P 2006 Appl. Phys. Lett. 88163504

[43] Barrat J L and Bocquet L 1999 Phys. Rev. Lett. 824671

[44] Vinogradova O I, Bunkin N F, Churaev N V, Kiseleva O A, Lobeyev A V and Ninham B W 1995 J. Colloid Interface Sci. $\mathbf{1 7 3} 443$

[45] Yakubov G E, Butt H J and Vinogradova O I 2000 J. Phys. Chem. B 1043407

[46] Borkent B, Dammler S, Schonherr H, Vansco G and Lohse D 2007 Phys. Rev. Lett. 98204502

[47] Ishida N, Inoue T, Miyahara M and Higashitani K 2000 Langmuir 166377

[48] Bonaccurso E, Butt H J and Craig V S J 2003 Phys. Rev. Lett. 90144501

[49] Granick S, Zhu Y and Lee H 2003 Nat. Mater. 2221

[50] Zhu Y X and Granick S 2002 Phys. Rev. Lett. 88106102

[51] Sarkar K and Prosperetti A 1996 J. Fluid Mech. 316223

[52] Lecoq N, Anthore R, Cichocki B, Szymczak P and Feuillebois F 2004 J. Fluid Mech. 513247

[53] Kunert C and Harting J 2007 Phys. Rev. Lett. 99176001

[54] Ybert C, Barentin C, Cottin-Bizonne C, Joseph P and Bocquet L 2007 Phys. Fluids 19123601

[55] Belyaev A V and Vinogradova O I 2010 J. Fluid Mech. $\mathbf{6 5 2} 489$ 
[56] Cottin-Bizonne C, Barentin C, Charlaix E, Bocquet L and Barrat J L 2004 Eur. Phys. J. E 15427

[57] Cottin-Bizonne C, Barrat J L, Bocquet L and Charlaix E 2003 Nat. Mater. 2237

[58] Hyväluoma J and Harting J 2008 Phys. Rev. Lett. 100246001

[59] Davis A M J and Lauga E 2009 Phys. Fluids 21011701

[60] Sbragaglia M and Prosperetti A 2007 Phys. Fluids 19043603

[61] Pirat C, Sbragaglia M, Peters A M, Borkent B M, Lammertink R G H, Wessling M and Lohse D 2008 Europhys. Lett. 816602

[62] Reyssat M, Yeomans J M and Quere D 2008 Europhys. Lett. 8126006

[63] Ajdari A 2002 Phys. Rev. E 65016301

[64] Stroock A D, Dertinger S K, Whitesides G M and Ajdari A 2002 Anal. Chem. 745306

[65] Ajdari A and Bocquet L 2006 Phys. Rev. Lett. 96186102

[66] Lauga E and Stone H A 2003 J. Fluid Mech. 48955

[67] Batchelor G K 1970 J. Fluid Mech. 44419

[68] Torquato S 2002 Random Heterogeneous Materials: Microstructure and Macroscopic Properties (Berlin: Springer)
[69] Feuillebois F, Bazant M Z and Vinogradova O I 2010 Phys. Rev. Lett. 104159902

[70] Stroock A D, Dertinger S K W, Ajdari A, Mezić I, Stone H A and Whitesides G M 2002 Science 295647

[71] Wang C Y 2003 Phys. Fluids 151114

[72] Stroock A D and McGraw G J 2004 Phil. Trans. R. Soc. A 362971

[73] Ou J, Moss J M and Rothstein J P 2007 Phys. Rev. E 76016304

[74] Feuillebois F, Bazant M Z and Vinogradova O I 2010 Phys. Rev. E 82055301

[75] Belyaev A V and Vinogradova O I 2010 Soft Matter 64563

[76] Reynolds O 1886 Phil. Trans. R. Soc. A 177157

[77] Joly L, Ybert C, Trizac E and Bocquet L 2004 Phys. Rev. Lett. 93257805

[78] Muller V M, Sergeeva I P, Sobolev V D and Churaev N V 1986 Colloid J. USSR 48606

[79] Squires T M 2008 Phys. Fluids 20092105

[80] Bahga S S, Vinogradova O I and Bazant M Z 2010 J. Fluid Mech. 644245

[81] Huang D M, Cottin-Bizzone C, Ybert C and Bocquet L 2008 Phys. Rev. Lett. 101064503 